Review Article

\title{
Effectiveness of Cotreatment with Kuntai Capsule and Climen for Premature Ovarian Failure: A Meta-Analysis
}

\author{
Qianwen Ma $\mathbb{D}^{1,2}$ Yong Tan $\mathbb{D}^{2},{ }^{2}$ and Genlin $M o \mathbb{D}^{3}$ \\ ${ }^{1}$ Gynecology Department, Zhenjiang Hospital Affiliated to Nanjing University of Chinese Medicine (Zhenjiang Hospital \\ of Traditional Chinese Medicine), Zhenjiang, China \\ ${ }^{2}$ Reproductive Medicine Department, Affiliated Hospital of Nanjing University of Chinese Medicine, Nanjing, China \\ ${ }^{3}$ Advanced Manufacturing Institution, Jiangsu University, Zhenjiang, China \\ Correspondence should be addressed to Yong Tan; nzyszy@163.com and Genlin Mo; 550402699@qq.com
}

Received 28 July 2019; Accepted 31 January 2020; Published 20 February 2020

Academic Editor: Carmen Mannucci

Copyright (c) 2020 Qianwen Ma et al. This is an open access article distributed under the Creative Commons Attribution License, which permits unrestricted use, distribution, and reproduction in any medium, provided the original work is properly cited.

\begin{abstract}
Objective. To compare the treatment efficacy of Kuntai capsule with Climen only in the therapy of premature ovarian failure. Methods. Randomized controlled trials were electronically retrieved from PubMed, Cochrane Library, Web of science, CBM, CNKI, Wanfang, and Weipu database. In addition, some related papers were manually checked. All papers were assessed according to the Cochrane Handbook for Systematic Reviews of Interventions, and the effective data were analyzed by Revman 5.3 Software. Results. 11 randomized control trials involving 1068 patients were included. Results of meta-analysis showed that $\mathrm{E}_{2}$ (estrogen), the total therapeutic effective rate of the group of Kuntai capsule, and hormone were higher than hormone only. The LH (luteinizing hormone), FSH (follicle-stimulating hormone), and Kupperman score of the group of Kuntai capsule and Climen were lower than Climen only. Conclusion. Available evidence shows that Kuntai capsule with Climen is more effective than Climen in the therapy of premature ovarian failure. Nowadays, the quality of the research studies is low. More large-scaled randomized trials will need to be carried out.
\end{abstract}

\section{Introduction}

In recent years, with the economic development, social progress, increased mental stress day by day, and the adverse living habits lead to a sharp increase in premature ovarian failure (POF) patients $[1,2]$. Premature ovarian failure refers to the ovarian function declining of women before 40 years old, belonging to the polymorphic diseases $[3,4]$. The main performance of the patients is low estrogen, reduced leucorrhea, amenorrhea, libido, infertility, and emotional abnormal fluctuations. In addition, the patients also suffer from the elevated level of FSH and LH, reduced $\mathrm{E}_{2}$ level, and elevated gonadotropin concentration. It may be clinically related to genetic, immune, congenital absence of enzyme, infection, environment, and iatrogenic factors [2,5-13]. Currently, the main treatment for this disease is hormone therapy, which can improve the patients' hormone levels and relieve symptoms [14-16]. However, long-term use of hormone drugs has great side effects, which can stimulate reproductive organs and increase the risk of endometrial cancer. In recent years, traditional Chinese medicine has been gradually applied in the treatment of premature ovarian failure, and its advantages are gradually highlighted. Some scholars have proposed that Climen combined with traditional Chinese medicine can improve the treatment effect and ovarian function, and at the same time, the side effects are small and the medication safety is good [17-27]. In view of this, the purpose of this study is to conduct a meta-analysis of the current clinical evaluation results of Kuntai capsule combined with Climen for premature ovarian failure and to preliminarily evaluate the advantages and disadvantages of Kuntai capsule combined with Climen in the treatment of premature ovarian failure. 


\section{Methods}

2.1. Inclusion and Exclusion Criteria. We included randomized controlled trials of Kuntai capsule with Climen compared with Climen in women with premature ovarian failure. We included trials no matter where she came from. We eliminated wrong, incomplete, and repeatable articles. We also eliminated the articles of the experimental group which did not use Kuntai capsule with Climen and the control group which did not use Climen monotherapy.

2.2. Intervention and Outcome Indicators. The experimental group was Kuntai capsule with Climen. The control group was Climen only. Outcome indicators included the total therapeutic effective rate, LH, FSH, $\mathrm{E}_{2}$ level, Kupperman score, and safety evaluation.

2.3. Systematic Search for Evidence. The databases of PubMed, Cochrane Library, Web of science, CBM, CNKI, Wanfang, and Weipu were searched from computer to identify relevant RCTs. We also performed a hand search to identify any other articles. The following search terms were used: Kuntai capsule, Kuntai, hormone, estradiol, Climen, progesterone, artificial period, artificial cycle, and premature ovarian failure. The trials should be selected with no restriction.

2.4. Data Extraction and Quality Appraisal of the Evidence. Two independent reviewers extracted the data according to the inclusion criteria. If the two reviewers disagreed, the difference was solved through discussion. If a consensus could not be reached, a third reviewer was consulted. Reviewers evaluated the evidence according to the Cochrane system evaluation member handbook on the quality evaluation criteria of RCT [28]: (1) which random allocation method to choose; (2) whether the trial was allocation concealment; (3) whether the trial used the blinding method; (4) whether there was incomplete data bias; (5) whether there was selective bias; (6) other bias.

2.5. Data Analysis. All meta-analyses were done in Revman 5.3 provided by the Cochrane Collaboration. The results were reported as odds ratios (ORs), with 95\% confidence interval (95\% CI) for dichotomous outcomes, and weighted mean difference (WMD) with $95 \%$ CI for continuous outcomes. The chi-square test was used to test heterogeneity across studies. Data were analyzed with a fixed effect model if no statistical heterogeneity was observed $\left(I^{2} \leqq 50 \%\right)$. Data were analyzed with a random effect model if statistical heterogeneity was observed $\left(I^{2}>50 \%\right)$. In the presence of heterogeneity, the two researchers checked the data entered and explored the variation by conducting sensitivity analysis. Publication bias was examined by the funnel plot. We used the $Z(u)$-test to compute statistics. According to the $Z$ $(u)$, the statistic $P$ was obtained. If $P<0.05$, there was statistical significance; If $P>0.05$, there was no statistical significance.

\section{Results}

3.1. Included Trials. Searching from each database, we received 192 citations, including 63 in CNKI, 50 in CBM, 51 in Wanfang, 28 in Weipu, 0 in Medline, 0 in Cochrane Library, and 0 in Web of science. Screening the citations, the 178 citations were ruled out (including repeating between libraries and the content which had nothing to do with the study). Then, 14 articles were obtained. By reading the full text, 3 articles were ruled out because they did not meet the inclusion criteria. Finally, 11 articles were included (Figure 1).

3.2. Methodological Quality. This study included 11 articles, a total of 1068 patients, which were randomized controlled trials in China. This research showed that there were 10 articles reporting the $\mathrm{FSH}$ and $\mathrm{E}_{2}$ level. 5 articles reported the LH level. 9 articles reported on the total therapeutic effective rate. 5 articles reported on the Kupperman score. 2 articles reported the safety analysis. See Table 1 for a summary of key details of these studies.

3.3. Analysis of the Level of $L H$. The meta-analysis results showed that the difference among the 5 groups was statistically significant on the level of LH: the combined effect $\mathrm{MD}=-7.01, \quad 95 \% \quad \mathrm{CI} \quad[-10.77,-3.24], \quad Z=3.65$, and $P=0.0003$. So the level of LH of Kuntai capsule with the hormone group was lower than the hormone group (Figure 2).

3.3.1. Sensitivity Analysis. This study analyzed the sensitivity of heterogeneity of the 5 articles. Getting rid of Yuan HF's article decreased the heterogeneity obviously, so it was likely to be the main source of heterogeneity.

3.4. Analysis of the Level of FSH. The meta-analysis results showed that the difference among the 10 groups was statistically significant on the level of FSH: the combined effect $\mathrm{MD}=-8.98, \quad 95 \% \quad \mathrm{CI}[-11.84, \quad-6.12], \quad Z=6.15$, and $P<0.00001$. So the level of FSH of Kuntai capsule with the hormone group was lower than the hormone group (Figure 3).

3.4.1. Sensitivity Analysis. This study analyzed the sensitivity of heterogeneity of the 10 articles. Getting rid of Yuan HF's article decreased the heterogeneity obviously, so it was likely to be the main source of heterogeneity.

3.5. Analysis of the Level of $E_{2}$. The meta-analysis results showed that the difference among the 10 groups was statistically significant on the level of $E_{2}$ : the combined effect $\mathrm{MD}=11.38,95 \% \mathrm{CI}[7.11,15.64], Z=5.23$, and $P<0.00001$. So the level of $E_{2}$ of Kuntai capsule with the hormone group was higher than the hormone group (Figure 4). 


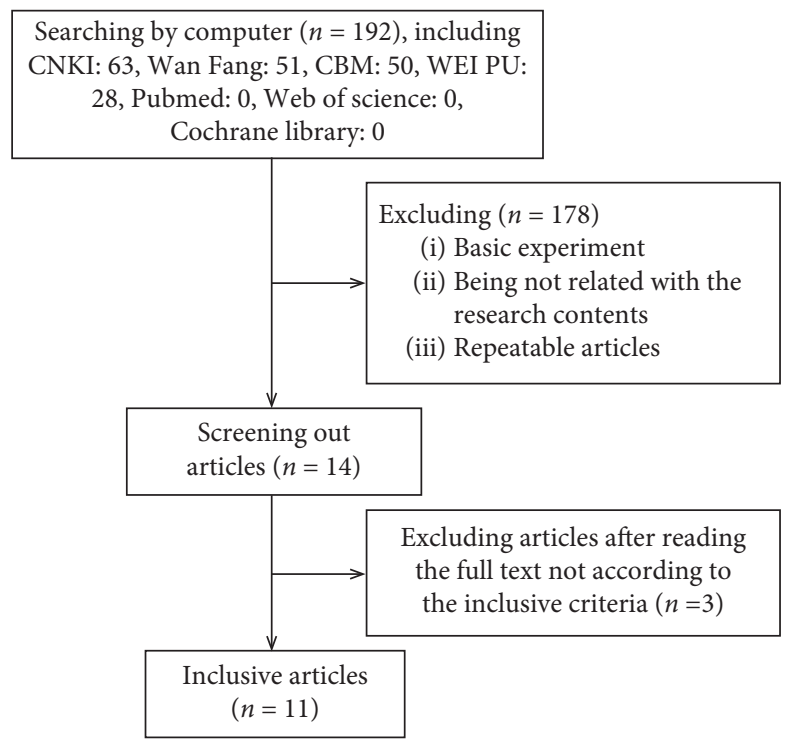

FIgURE 1: Data screening process.

TABle 1: Summary of clinical trials examining the effects of Kuntai capsule with Climen on patients with POF.

\begin{tabular}{|c|c|c|c|c|c|}
\hline Studies & Participants & Treatment group & $\begin{array}{l}\text { Control } \\
\text { group }\end{array}$ & Main results & $\begin{array}{l}\text { Course of } \\
\text { treatment }\end{array}$ \\
\hline $\mathrm{Xu}[17]$ & $N=140$ & $\begin{array}{c}\text { Climen + Kuntai capsule } \\
\qquad N=70\end{array}$ & $\begin{array}{l}\text { Climen } \\
N=70\end{array}$ & $\begin{array}{c}\text { Treatment group had a better } \\
\text { effect }\end{array}$ & 6 months \\
\hline Xing [18] & $N=140$ & $\begin{array}{c}\text { Climen + Kuntai capsule } \\
\qquad N=70\end{array}$ & $\begin{array}{l}\text { Climen } \\
N=70\end{array}$ & $\begin{array}{c}\text { Treatment group had a better } \\
\text { effect }\end{array}$ & 6 months \\
\hline Yang [19] & $N=126$ & $\begin{array}{c}\text { Climen + Kuntai capsule } \\
\qquad N=63\end{array}$ & $\begin{array}{l}\text { Climen } \\
N=63\end{array}$ & $\begin{array}{c}\text { Treatment group had a better } \\
\text { effect }\end{array}$ & 6 months \\
\hline Yuan and $\mathrm{Hu}[20]$ & $N=80$ & $\begin{array}{c}\text { Climen }+ \text { Kuntai capsule } \\
\qquad N=40\end{array}$ & $\begin{array}{l}\text { Climen } \\
N=40\end{array}$ & $\begin{array}{c}\text { Treatment group had a better } \\
\text { effect }\end{array}$ & 3 months \\
\hline Chen [21] & $N=90$ & $\begin{array}{c}\text { Climen }+ \text { Kuntai capsule } \\
\qquad N=45\end{array}$ & $\begin{array}{l}\text { Climen } \\
N=45\end{array}$ & $\begin{array}{c}\text { Treatment group had a better } \\
\text { effect }\end{array}$ & 6 months \\
\hline Zhong et al. [22] & $N=70$ & $\begin{array}{c}\text { Climen }+ \text { Kuntai capsule } \\
\qquad N=35\end{array}$ & $\begin{array}{l}\text { Climen } \\
N=35\end{array}$ & $\begin{array}{c}\text { Treatment group had a better } \\
\text { effect }\end{array}$ & 3 months \\
\hline Dai [23] & $N=146$ & $\begin{array}{c}\text { Climen }+ \text { Kuntai capsule } \\
\qquad N=73\end{array}$ & $\begin{array}{l}\text { Climen } \\
N=73\end{array}$ & $\begin{array}{c}\text { Treatment group had a better } \\
\text { effect }\end{array}$ & 3 months \\
\hline Xiao et al. [24] & $N=90$ & $\begin{array}{c}\text { Climen }+ \text { Kuntai capsule } \\
\qquad N=45\end{array}$ & $\begin{array}{l}\text { Climen } \\
N=45\end{array}$ & $\begin{array}{c}\text { Treatment group had a better } \\
\text { effect }\end{array}$ & 3 months \\
\hline Liang [25] & $N=78$ & $\begin{array}{c}\text { Climen }+ \text { Kuntai capsule } \\
\qquad N=39\end{array}$ & $\begin{array}{l}\text { Climen } \\
N=39\end{array}$ & $\begin{array}{c}\text { Treatment group had a better } \\
\text { effect }\end{array}$ & 3 months \\
\hline Liu [26] & $N=60$ & $\begin{array}{c}\text { Climen }+ \text { Kuntai capsule } \\
N=30\end{array}$ & $\begin{array}{l}\text { Climen } \\
N=30\end{array}$ & $\begin{array}{c}\text { Treatment group had a better } \\
\text { effect }\end{array}$ & 6 months \\
\hline Huang [27] & $N=48$ & $\begin{array}{c}\text { Climen + Kuntai capsule } \\
\qquad N=23\end{array}$ & $\begin{array}{l}\text { Climen } \\
N=25\end{array}$ & $\begin{array}{c}\text { Treatment group had a better } \\
\text { effect }\end{array}$ & 3 weeks \\
\hline
\end{tabular}

3.5.1. Sensitivity Analysis. This study analyzed the sensitivity of heterogeneity of the 10 articles. Getting rid of Xiao's article decreased the heterogeneity obviously, so it was likely to be the main source of heterogeneity.

3.6. Analysis of the Therapeutic Effective Rate. The metaanalysis results showed that the difference among the 8 groups was statistically significant on the therapeutic effective rate: the combined effect $\mathrm{OR}=3.88,95 \%$ CI $[2.47$,
6.08], $Z=5.90$, and $P<0.0001$. So the therapeutic effective rate of Kuntai capsule with the hormone group was higher than the hormone group (Figure 5).

3.7. Analysis of the Kupperman Score. The meta-analysis results showed that the difference among the 5 groups was statistically significant on the Kupperman score. The combined effect $\mathrm{MD}=-3.86,95 \%$ CI $[-4.92,-2.80], Z=7.14$, $P<0.00001$. So the Kupperman score of Kuntai capsule with 


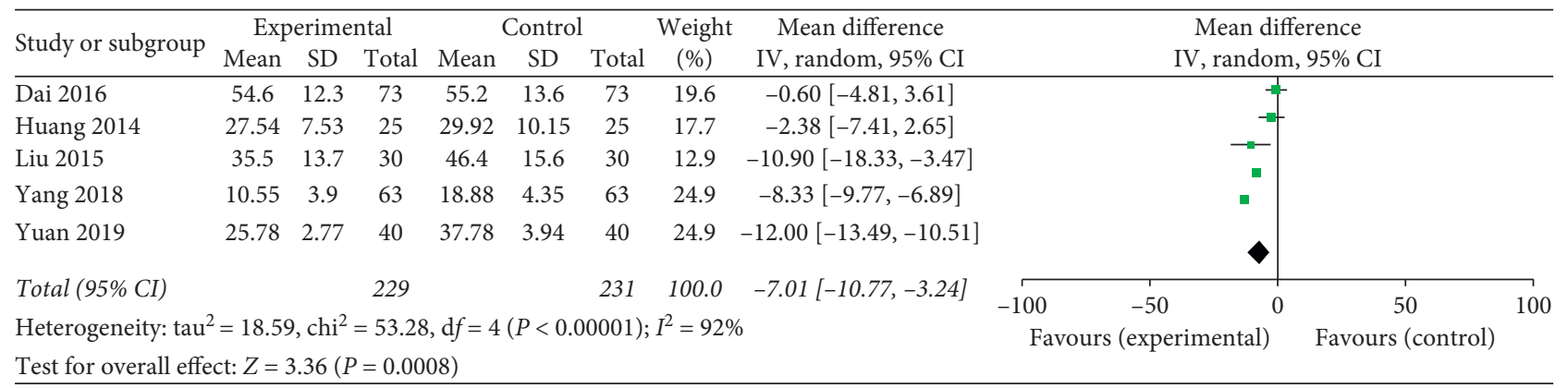

Figure 2: Comparison of the level of LH.

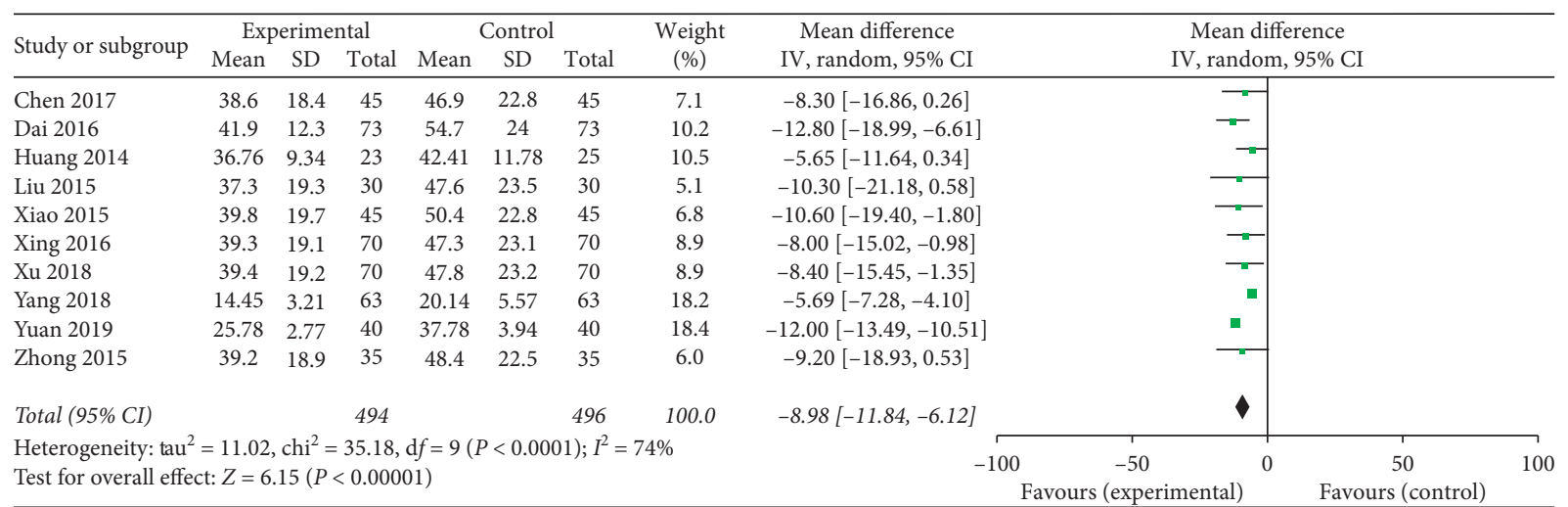

Figure 3: Comparison of the level of FSH.

\begin{tabular}{|c|c|c|c|c|c|c|c|c|c|c|}
\hline \multirow{2}{*}{ Study or subgroup } & \multicolumn{3}{|c|}{ Experimental } & \multicolumn{3}{|c|}{ Control } & \multirow{2}{*}{$\begin{array}{l}\text { Weight } \\
(\%)\end{array}$} & \multirow{2}{*}{$\begin{array}{l}\text { Mean difference } \\
\text { IV, random, 95\% CI }\end{array}$} & \multirow{2}{*}{\multicolumn{2}{|c|}{$\begin{array}{c}\text { Mean difference } \\
\text { IV, random, 95\% CI }\end{array}$}} \\
\hline & Mean & $\mathrm{SD}$ & Total & Mean & $\mathrm{SD}$ & Total & & & & \\
\hline Chen 2017 & 143.86 & 41.84 & 45 & 125.88 & 33.76 & 45 & 5.2 & $17.98[2.27,33.69]$ & & \\
\hline Dai 2016 & 124.5 & 15.2 & 73 & 109.6 & 12.8 & 73 & 14.7 & $14.90[10.34,19.46]$ & & - \\
\hline Huang 2014 & 94.37 & 21.45 & 23 & 81.64 & 19.35 & 25 & 7.7 & $12.73[1.14,24.32]$ & & \\
\hline Liu 2015 & 142.03 & 41.84 & 30 & 118.91 & 34.87 & 30 & 3.8 & $23.12[3.63,42.61]$ & & \\
\hline Xiao 2015 & 14.31 & 1.84 & 45 & 11.38 & 2.57 & 45 & 17.5 & $2.93[2.01,3.85]$ & & $\square$ \\
\hline Xing 2016 & 142 & 41.47 & 70 & 125.51 & 34.13 & 70 & 7.0 & $16.49[3.91,29.07]$ & & \\
\hline Xu 2018 & 142.4 & 41.84 & 70 & 126.25 & 34.5 & 70 & 6.9 & $16.15[3.45,28.85]$ & & \\
\hline Yan 2018 & 42.72 & 6.63 & 63 & 33.3 & 6.83 & 63 & 16.7 & $9.42[7.07,11.77]$ & & $=$ \\
\hline Yuan 2019 & 84.47 & 7.73 & 40 & 76.21 & 7.83 & 40 & 15.9 & $8.26[4.85,11.67]$ & & $-\pi$ \\
\hline Zhong 2015 & 143.13 & 37.8 & 35 & 127.35 & 33.4 & 35 & 4.8 & $15.78[-0.93,32.49]$ & & \\
\hline Total (95\% CI) & & & 494 & & & 496 & 100.0 & $11.38[7.11,15.64]$ & & \\
\hline \multicolumn{8}{|c|}{$\begin{array}{l}\text { Heterogeneity: } \operatorname{tau}^{2}=26.84, \mathrm{chi}^{2}=69.21, \mathrm{~d} f=9(P<0.0001) ; I^{2}=87 \% \\
\text { Test for overall effect: } Z=5.23(P<0.00001)\end{array}$} & & $\begin{array}{l}-100-50 \\
\text { Favours (experimental) }\end{array}$ & $\begin{array}{cc}50 & 100 \\
\text { Favours (control) }\end{array}$ \\
\hline
\end{tabular}

Figure 4: Comparison of the level of $\mathrm{E}_{2}$.

the Climen group was lower than the Climen group (Figure 6).

3.7.1. Sensitivity Analysis. This study analyzed the sensitivity of heterogeneity of the 5 articles. Getting rid of Dai SM's article decreased the heterogeneity obviously, so it was likely to be the main source of heterogeneity.

3.8. Safety Analysis and Publication Bias. Only two articles presented adverse effects of their studies, and the descriptions were not very detailed. Thus, we were unable to analyze the safety outcomes. In this study, inverted funnel graph analysis was performed on the inverse of standard error of
OR of LH, FSH, $\mathrm{E}_{2}$ level, total therapeutic effective rate, and Kupperman score. The asymmetrical pattern suggested small sample studies and possible publication bias, as shown in Figures 7-11.

\section{Discussion}

The results of this study showed that the total therapeutic effective rate, $\mathrm{LH}, \mathrm{FSH}, \mathrm{E}_{2}$ level, Kupperman score of Kuntai capsule, and Climen group were more effective than those of the Climen group in the treatment of premature ovarian failure. POF will not only cause the reduction of estrogen level, but also lead to the loss of female fertility in severe cases, which will exert great pressure on 


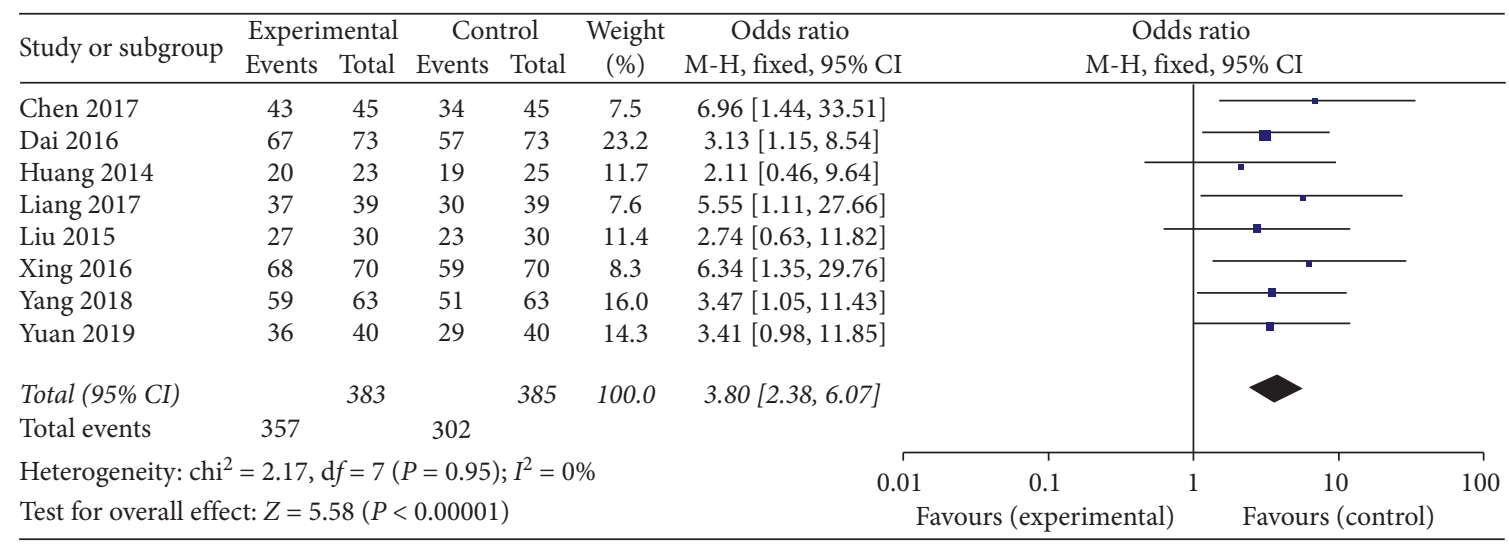

FIgURE 5: Comparison of the therapeutic effective rate.

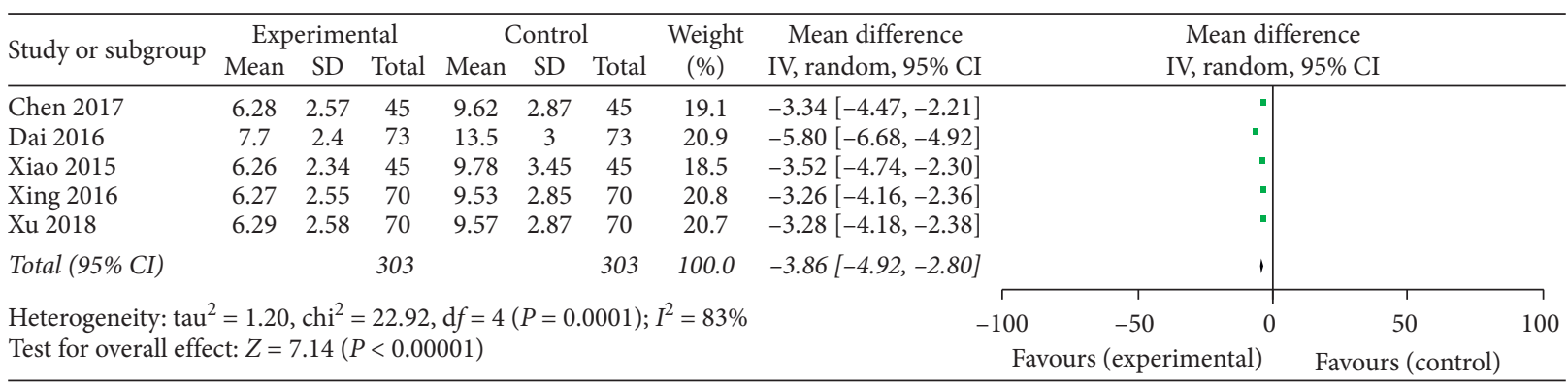

FIgURE 6: Comparison of the Kupperman score.

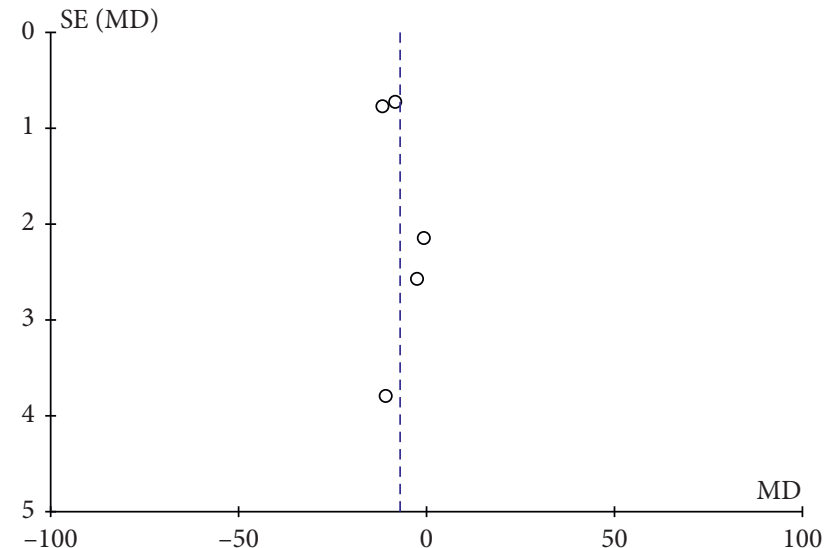

Figure 7: Funnel plot of the level of LH.

female physiology and psychology and affect the quality of life. Climen [29, 30] (estradiol valerate tablets/estradiol cyprogesterone tablets) is the commonly used Western medicine treatment of POF, which can simulate the sex hormone levels of the menstrual cycle in the body and induce menstrual cramps. But long-term use of Climen may lead to water sodium retention patients and increase the risk of endometrial cancer and breast cancer. Kuntai capsule [31-37] originates from Rhizoma coptidis and donkey-hide gelatin soup of Treatise on Febrile and Miscellaneous Disease, mainly composed of Rhizoma

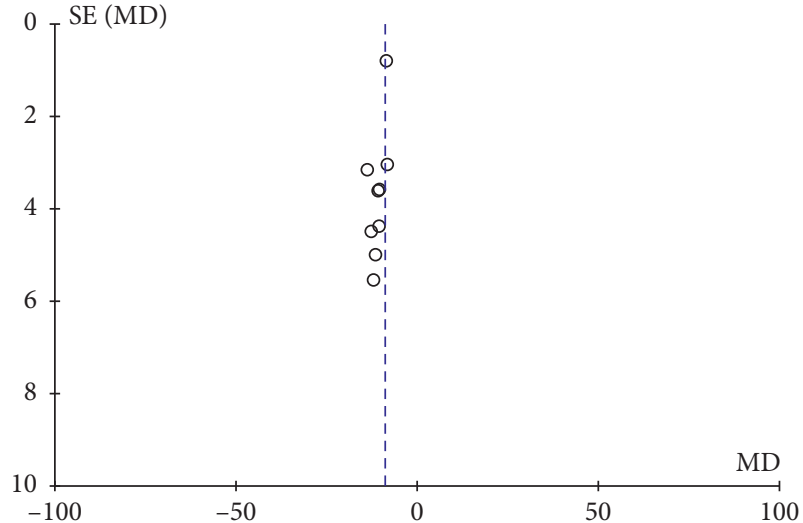

FIgURE 8: Funnel plot of the level of FSH.

coptidis, donkey-hide gelatin, radix paeoniae alba, Poria cocos, Radix scutellariae, and Rehmannia glutinosa. It can improve the symptoms such as amenorrhea, hypomenorrhea, bradymenorrhea, night sweats, and lumbar debility. Therefore, Kuntai capsule combined with Climen can combine the advantages of the two therapy methods and make the female reproductive environment more harmonious.

The methodological quality evaluation results of the included researches showed that there were many lowquality studies. The clinical studies included in this study had methodological problems such as randomization, blind 


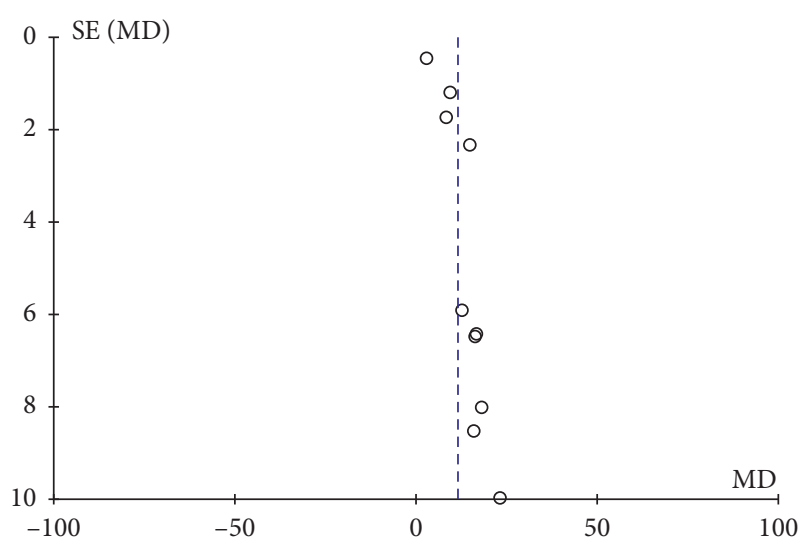

Figure 9: Funnel plot of the level of $\mathrm{E}_{2}$.

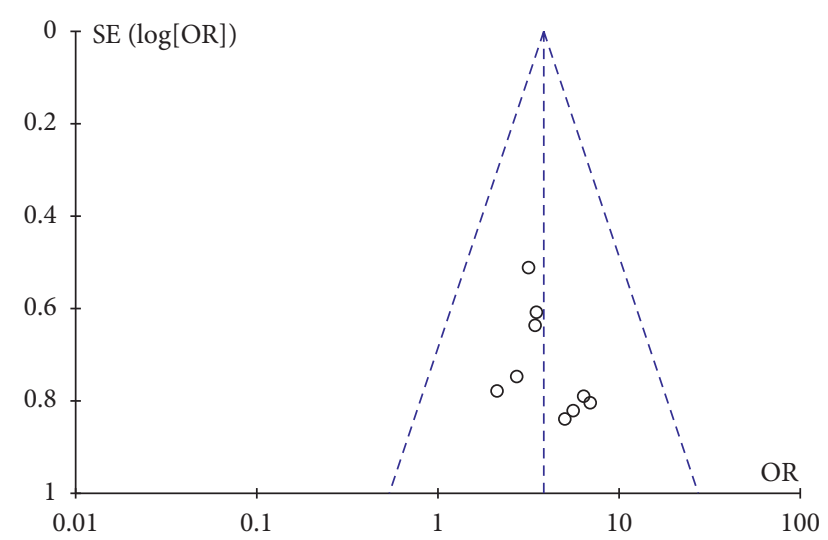

FIgURE 10: Funnel plot of the total therapeutic effective rate.

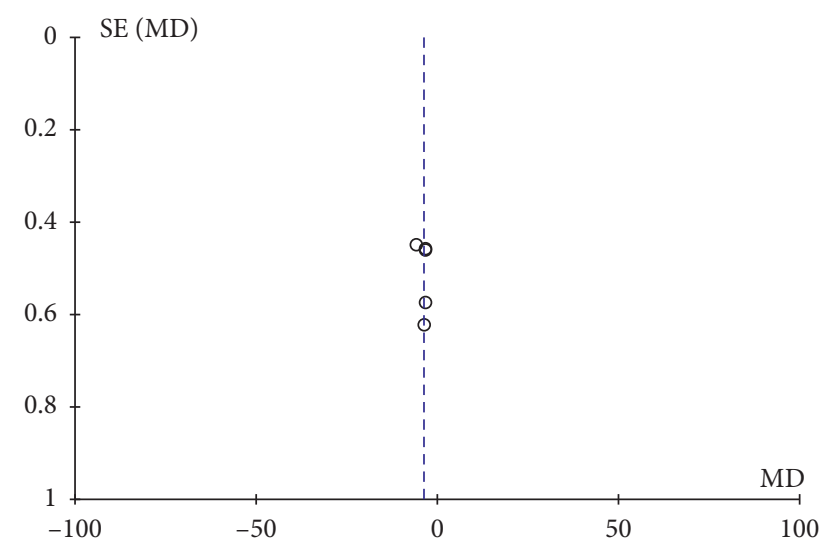

FIGURE 11: Funnel plot of the Kupperman score.

method, and follow-up. All of these could lead to the occurrence of bias and affect the accuracy and reliability of the trials. Among the 11 references included in this metaanalysis, only 5 references described the specific randomized method (the random number table method or treatment order), although all the reports mentioned the random method. In most studies, there was no mention of the concealment of the random scheme, and no specific description of the blind method, loss of follow-up, and withdrawal cases, which would affect the demonstration strength of the study. Meanwhile, LH, FSH, E2 level, and total therapeutic effective rate were the main indicators for the evaluation of efficacy, and the safety analysis of drugs and Kupperman score was less concerned. In the future, randomized controlled trials should not only be designed reasonably, but also include large samples, rigorous randomized methods, and double-blind studies.

Therefore, this study considers that the combination of Kuntai capsule and Climen for premature ovarian failure has a better curative effect. However, it needs to be supported by more rigorous double-blind randomized controlled trials with a larger sample to ensure more convinced research results and provide more reliable evidence for the combination of Kuntai capsule and Climen for premature ovarian failure.

\section{Conflicts of Interest}

The authors declare that they have no conflict of interest.

\section{Acknowledgments}

This research was supported by the Natural Science Foundation of China (no. 81873333 and 81373683), and Zhenjiang Health and Family Planning Science Technology Key Project (no. SHW2017016).

\section{References}

[1] K. Maclaran and N. Panay, "Premature ovarian failure," Przeglad Menopauzalny, vol. 37, no. 1, pp. 51-56, 2017.

[2] K. Jankowska, "Premature ovarian failure," Menopausal Review, vol. 16, no. 2, pp. 51-56, 2017.

[3] R. Melekoglu, O. Ciftci, S. Eraslan et al., "Beneficial effects of curcumin and capsaicin on cyclophosphamide-induced premature ovarian failure in a rat model," Journal of Ovarian Research, vol. 11, no. 1, p. 33, 2018.

[4] R. Asadi, M. D. Omrani, H. Ghaedi et al., "Premutations of FMR1 CGG repeats are not related to idiopathic premature ovarian failure in Iranian patients: a case control study," Gene, vol. 676, pp. 189-194, 2018.

[5] V. P. Chernyshov, T. V. Radysh, I. V. Gura et al., "Immune disorders in women with premature ovarian failure in initial period," American Journal of Reproductive Immunology, vol. 46, no. 3, pp. 220-225, 2015.

[6] M. S. Yucebilgin, M. C. Terek, A. Ozsaran et al., "Effect of chemotherapy on primordial follicular reserve of rat: an animal model of premature ovarian failure and infertility," Australian \& new Zealand Journal of Obstetrics \& Gynaecology, vol. 44, no. 1, pp. 6-9, 2015.

[7] E. Kovanci and A. K. Schutt, "Premature ovarian failure," Obstetrics and Gynecology Clinics of North America, vol. 42, no. 1, pp. 153-161, 2015.

[8] Z. Wang, Y. Wang, T. Yang et al., "Study of the reparative effects of menstrual-derived stem cells on premature ovarian failure in mice," Stem Cell Research \& Therapy, vol. 8, no. 1, p. 11, 2017.

[9] Y. M. Van Kasteren, M. Von Blomberg, C. De Koning et al., "Incipient ovarian failure and premature ovarian failure show the same immunological profile," American Journal of Reproductive Immunology, vol. 43, no. 6, pp. 359-366, 2015. 
[10] L. Jing, X. L. Li, S. Hui et al., "A general description for Chinese medicine in treating premature ovarian failure," Chinese Journal of Integrative Medicine, vol. 23, no. 2, pp. 91-97, 2017.

[11] Z. Yan, Y. Dai, H. Fu et al., "Curcumin exerts a protective effect against premature ovarian failure in mice," Journal of Molecular Endocrinology, vol. 60, no. 3, 2018.

[12] Y. I. Mahmoud, "Chronic cholestasis is associated with hypogonadism and premature ovarian failure in adult rats (cholestasis causes ovarian hypogonadism)," Ultrastructural Pathology, vol. 42, no. 1, pp. 23-31, 2018.

[13] R. Kumar, M. Alwani, S. Kosta et al., "BMP15 and GDF9 gene mutations in premature ovarian failure," Journal of Reproduction \& Infertility, vol. 18, no. 1, pp. 185-189, 2017.

[14] H. Medenvrtovec, A. Levičnik, A. Ihan et al., "Autoimmune aspects of premature ovarian failure," Zdravniški Vestnik, vol. 78, pp. 151-156, 2018.

[15] A. Eslami, K. Farahmand, M. Totonchi et al., "FMR1 premutation: not only important in premature ovarian failure but also in diminished ovarian reserve," Human Fertility, vol. 20, no. 2, pp. 120-125, 2017.

[16] E. C. Somers, W. Marder, G. M. Christman et al., "Use of a gonadotropin-releasing hormone analog for protection against premature ovarian failure during cyclophosphamide therapy in women with severe lupus," Arthritis Rheum, vol. 52, no. 9, pp. 2761-2767, 2014.

[17] H. Xu, "Clinical analysis of treatment of premature ovarian failure by Climen and Kuntai capsule," Women's Health Research, vol. 12, p. 59, 2018.

[18] Y. Xing, "Observation on the curative effect of Kuntai capsule + Climen in the treatment of premature ovarian failure," China Foreign Medical Treatment, vol. 35, no. 18, pp. 111-112, 2016.

[19] X. Yang, "Clinical effect observation of Kuntai capsule combined with estrogen and progesterone in the treatment of premature ovarian failure," Henan Medical Research, vol. 27, no. 12, pp. 129-130, 2018.

[20] H. Yuan and Y. Hu, "Effect of Kuntai capsule combined with hormone replacement therapy on premature ovarian failure," Hubei Journal of TCM, vol. 41, no. 1, pp. 16-18, 2019.

[21] J. Chen, "Efficacy of estradiol valerate tablets/estradiol cyprogesterone tablets combined with Kuntai capsules in the treatment of patients with premature ovarian failure," Medical Equipment, vol. 30, no. 8, pp. 92-93, 2017.

[22] P. Zhong, L. Yao, Y. Wu et al., "Effect analysis on treatment of premature ovarian failure with Climen tablet combined with Kuntai capsule," Contemporary Medicine Forum, vol. 13, no. 8, pp. 148-149, 2015.

[23] S. Dai, "Climen combined with Kuntai capsule for the treatment of premature ovarian failure," Journal of Practical Gynecologic Endocrinology (Electronic Edition), vol. 3, no. 12, pp. 51-52, 2016.

[24] P. Xiao, Y. Xu, and Y. Shi, "Observation on the curative effect of Climen combined with Kuntai capsule in the treatment of premature ovarian failure," Chinese Journal of General Practice, vol. 13, no. 5, pp. 774-775, 2015.

[25] W. Liang, "Effect analysis of Climen tablet combined with Kuntai capsule in the treatment of premature ovarian failure," Henan Medical Research, vol. 26, no. 8, pp. 1426-1427, 2017.

[26] Q. Liu, "Climen and Kuntai capsule for clinical observation of premature ovarian failure," Shenzhen Journal of Integrated Traditional Chinese and Western Medicine, vol. 25, no. 9, pp. 81-82, 2015.
[27] A. Huang, "Effect of Kuntai capsule combined with Climen on premature ovarian failure and the effect of related sex hormones," Guiding Journal of Traditional Chinese Medicine and Pharmacy, vol. 20, no. 15, pp. 73-74, 2014.

[28] The Cochrane Collaboration, Cochrane Handbook for Systematic Reviews of Interventions Version 5.1.0, Cochrane, London, UK, 2011, http://www.cochrane.org/training/ cochrane-handbook.

[29] H. P. G. Schneider, H. P. Rosemeier, J. Schnitker, S. Gerbsch, and R. Turck, "Application and factor analysis of the menopause rating scale (MRS) in a post-marketing surveillance study of Climen," Maturitas, vol. 37, no. 2, pp. 113-124, 2000.

[30] H. Zhang, Y. Chu, P. Zhou et al., "Dehydroepiandrosterone plus climen supplementation shows better effects than dehydroepiandrosterone alone on infertility patients with diminished ovarian reserve of low-FSH level undergoing invitro fertilization cycles: a randomized controlled trial," $R e$ productive Biology \& Endocrinology, vol. 14, no. 1, p. 9, 2016.

[31] Q. Y. Zhang, J. W. Wang, and J. M. Yu, "Efficacy and safety of Kuntai capsule and estrogen for menopausal syndrome: a meta-analysis," Zhonghua Yi Xue Za Zhi, vol. 93, no. 43, pp. 3445-3449, 2013.

[32] D. M. Shi and G. Z. Sun, "Clinical observation of Kuntai capsule on female menopause insomnia associated with anxiety and depression," Chinese Traditional \& Herbal Drugs, vol. 44, no. 24, pp. 3531-3533, 2013.

[33] J. Zhang, L. L. Gong, and S. F. Zhang, "Effects of kuntai capsule on quality of life, breast distending pain and vaginal bleeding in women at early stage of menopause," Chinese Journal of Integrated Traditional \& Western Medicine, vol. 28, no. 11, pp. 972-976, 2008.

[34] W. Li, "Effects of Kuntai Capsule and hormone replacement therapy on cognitive function and mental symptoms of early postmenopausal women: a randomized controlled trial," Journal of Chinese Integrative Medicine, vol. 8, no. 4, pp. 321-327, 2010.

[35] Z. Ruihua, X. Shuwu, L. Guoting et al., "Hormone-like activities of Kuntai capsule in the uteri of ovariectomized rats and immature rabbits," Journal of Traditional Chinese Medicine, vol. 38, no. 6, pp. 55-63, 2018.

[36] B. Zhang, C. Nan, X. M. Qiu et al., "Effects of Heyan Kuntai capsule on follicular evelopment and oocyte cohesin levels in aged mice," Chinese Journal of Integrative Medicine, pp. 1-9, 2018.

[37] Q. Zhou, J. Tao, H. Song et al., "Chinese herbal medicine Kuntai capsule for treatment of menopausal syndrome: a systematic review of randomized clinical trials," Complementary Therapies in Medicine, vol. 29, pp. 63-71, 2016. 